\title{
Hybrid Modulation Scheme Combining PPM with Differential Chaos Shift Keying Modulation
}

\author{
Meiyuan Miao, Lin Wang, Senior Member, IEEE, Marcos Katz, Member, IEEE and Weikai Xu, Member, IEEE
}

\begin{abstract}
In conventional $M$-ary differential chaos shift keying modulation (DCSK) systems, the distance between constellation points gets closer as $M$ increases, resulting in poor performance. A hybrid modulation scheme based on pulse position modulation (PPM) and DCSK is proposed in the paper to improve bit-error-rate (BER) performance. In this scheme, one part of the bits is modulated by PPM while the other part is modulated by DCSK. Thus, information bearing signals are simultaneously modulated by the information bit and the selected pulse position of PPM which is determined by extra information bits. Analytical bit-error-rate (BER) performance of the proposed scheme is derived and verified by simulations. Results show that the considered scheme outperforms conventional M-DCSK, code index modulation DCSK (CIM-DCSK) and commutation code index DCSK (CCI-DCSK) in additive white Gaussian noise (AWGN) and multipath Rayleigh fading channels.
\end{abstract}

Index Terms-Differential chaos shift keying modulation(DCSK), pulse position modulation(PPM), hybrid modulation, bit error rate (BER).

\section{INTRODUCTION}

Chaotic communication has gained increasing attention as it can be used widely in spread spectrum communication systems due to its low-power, low complexity, and excellent anti-fading capabilities [1], [2]. Differential chaos shift keying (DCSK), a modulation scheme proposed for chaotic communications [3], is characterized by its simple transceiver configuration and excellent performance over multipath fading channels [4], only requiring a simple non-coherent demodulator without channel estimators and equalization [5]. Some variants of DCSK, such as permutation index DCSK(PI-DCSK) [6] and code index modulation DCSK (CIM,CCI-DCSK) [1] [7] which used index modulation [8][9] have also been proposed. A multi-resolution $M$-ary DCSK with $M$-ary phase-shiftkeying (MPSK) constellation is proposed based on quadrature chaotic shift keying (QCSK) [10] which offers better BER performance by changing the structure of constellation.

Due to its simplicity and high performance, pulse position modulation (PPM) is also widely used in spread spectrum communications. The idea of a hybrid combining modulation using PPM was first proposed in optical communications [11]. A hybrid pulse PPM-BPSK add space for transmitted reference pulse cluster (TRPC) is proposed to improve performance in [12]. Similarly, DCSK also can be combined with PPM

M. Miao, L. Wang and W. Xu are with the Department of Communication Engineering, Xiamen University, Xiamen, 361005, P. R. China. L. Wang is the corresponding author (e-mail: meiyuanmiao@foxmail.com; wanglin@xmu.edu.cn; xweikai@xmu.edu.cn).

M. Katz is with the Centre for Wireless Communications, University of Oulu, Oulu 90014, Finland (e-mail:marcos.katz@ee.oulu.fi). for better performance in spread spectrum communication systems.

To improve the BER performance of conventional $M$-ary DCSK, a hybrid PPM-DCSK modulation scheme is proposed in this letter. The main contribution of the brief are summarized as follows. Firstly, based on a hybrid two-dimensional modulation scheme, a mixed modulation scheme with PPM and DCSK is proposed which the PPM parts bearing $m_{c}$ bits signal. Secondly, the bit error rate (BER) expression for the considered system is obtained analytically and validated then by simulations. Results show that the proposed system has better BER performance than conventional M-DCSK, multidimensional CIM-DCSK and CCI-DCSK schemes.

The paper is organized as follows. Section II presents the system model of PPM-DCSK system. The calculation of the BER expressions of the proposed system is derived in section III. Simulation results and discussions are presented in section IV. Section V concludes the paper.

\section{SySTEM MOdeL}

The transmitter of proposed system is shown in Fig. 1, where the total transmitted bits are $m_{c}+1$ and symbol duration is $\left(2^{m_{c}}+1\right) R$. In the proposed system, $m_{c}$ bits are mapped into a PPM position. The transmitted signal $s_{l}$ can be expressed as

$$
\mathbf{s}_{\mathbf{l}}=[\underbrace{\mathbf{c}_{x}}_{\text {reference }}, \underbrace{b_{l} \mathbf{s}_{P P M} \bigotimes \mathbf{c}_{x}}_{\text {information-bearing }}]
$$

where $\mathbf{c}_{x}$ is a $R$-length chaotic signal, $b_{l} \in\{-1,1\}$ is information bit, $\otimes$ is Kronecker operator. In the proposed system, the information bit is transmitted on one position in the PPM frame. The position is determined by mapping bits. $\mathbf{s}_{P P M}=\left[0,0, \ldots 1_{a_{l}}, \ldots, 0\right]_{1 \times P}\left(P=2^{m_{c}}\right)$ in the PPM signal, where $1_{a_{l}}$ represents that the $a_{l}^{t h}$ position of $\mathbf{s}_{P P M}$ is 1 . $a_{l}$ is a position index modulation symbol which is converted by mapping bits.

Assuming that the transmitted signal is corrupted by a multipath Rayleigh fading channel, the received signal can be written as

$$
\mathbf{r}_{l}=\sum_{l=1}^{L} \alpha_{l} \delta\left(t-\tau_{l}\right) \otimes \mathbf{s}_{l}+\mathbf{n}_{l},
$$

where $L$ is the number of paths, $\alpha_{l}$ and $\tau_{l}$ are the channel coefficient and the path delay of the lth path, respectively, $\otimes$ denotes the convolution operator. Moreover, the channel coefficients are constant over each symbol duration and the maximum multipath delay is much shorter than $R$, i.e., $R>>$ $\tau_{l \text { max }} . n_{l}$ is the additive white Gaussian noise (AWGN) with 


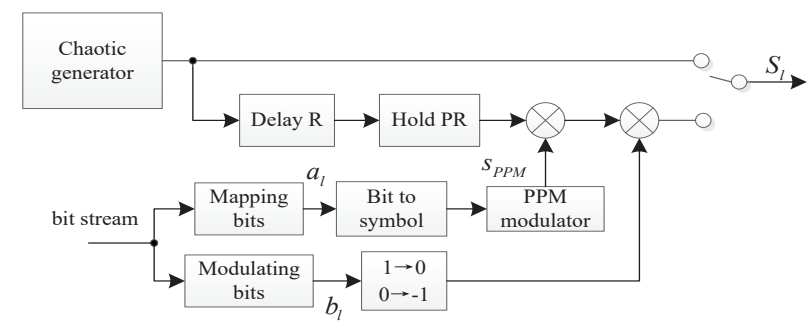

Fig. 1. Block diagram of PPM-DCSK transmitter.

zero mean value and variance of $N_{0} / 2$. The block diagram of the receiver is shown in Fig. 2. The receiver not only needs to detect the modulation bits from DCSK signal, but also the index position of PPM. That means the received reference signal need to be correlated with each $R$-length parts of received $\mathbf{s}_{P P M}$, thus the $a_{l}$ th part of information-bearing signal in PPM signal can be written as

$$
\mathbf{r}_{i n f}=\mathbf{r}_{a_{l}}, \quad 1 \leq a_{l} \leq P,
$$

The decision variable $I_{m}$ of the $m^{\text {th }}$ branch when $m=a_{l}$ can be expressed as

$$
I_{m}=\sum_{i=1}^{R}\left(\sum_{l=1}^{L} \alpha_{l} \mathbf{c}_{x}+\mathbf{n}_{r}\right)\left(\sum_{l=1}^{L} \alpha_{l} b_{l} \mathbf{c}_{x}+\mathbf{n}_{r-R},\right)
$$

Similarly, when $m \neq a_{l}$, the decision variable of the $m_{t h}$ branch can be expressed as

$$
\begin{aligned}
I_{m}^{\prime} & =\sum_{i=1}^{R}\left(\sum_{l=1}^{L} \alpha_{l} \mathbf{c}_{x}+\mathbf{n}_{r}\right)\left(\mathbf{n}_{r-R}\right) \\
& =\sum_{i=1}^{R} \sum_{l=1}^{L} \alpha_{l} \mathbf{c}_{x} \mathbf{n}_{r-R}+\sum_{i=1}^{R} \mathbf{n}_{r} \mathbf{n}_{r-R},
\end{aligned}
$$

where the symbol $a_{l}$ and $b_{l}$ can be estimated respectively as

$$
\begin{aligned}
& \hat{a}_{l}=\arg \max _{m=1, \ldots P}\left(\left|I_{m}\right|,\left|I_{m}^{\prime}\right|\right), \\
& \hat{b}_{l}=\operatorname{sign}\left(I_{\hat{a}_{l}}\right),
\end{aligned}
$$

it can be seen from (6) that the maximum of estimated $a_{l}$ is decided as the position of information bearing signal in PPM frame, and $b_{l}$ can be obtained by demodulating outputs of corresponding correlators. Then the bits mapped by position can be obtained from the decimal-to-binary converting part. For $m_{c}$ mapped bits, the symbol duration of PPM-DCSK, CIM-DCSK and CCI-DCSK is $\left(2^{m_{c}}+1\right) * R$ [2], $\left(2^{m_{c}}+1\right) * R$ and $2^{n} * R\left(n \geq m_{c}\right)$ [7], respectively. Thus they have similar bandwidth efficiency.

\section{PERFORMANCE OF PPM-DCSK ANALYSIS}

\section{A. System BER analysis}

The total system BER is a function of the BER of the modulated bits $P_{e m}$ and the BER of the mapped $P_{e c i m}$, which is the function of the error probability of PPM detection error probability $P_{e d}$. We set $m_{c}$ as the number of bits mapped to one position of PPM index modulating symbol. The symbol

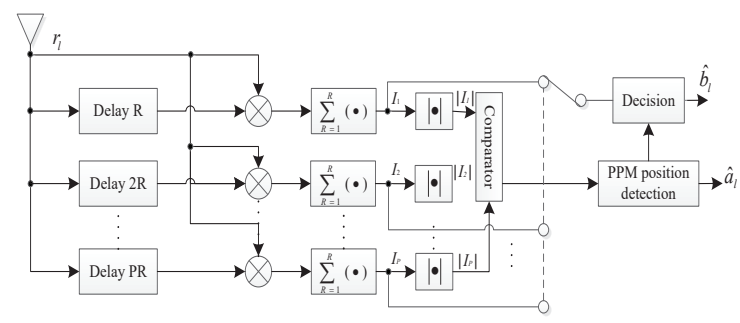

Fig. 2. Block diagram of PPM-DCSK receiver.

$a_{l}$ is mapped from $m_{c}$ bits and the probability of detecting one of the remaining $P-1$ incorrect positions are the same at all positions. Thus, the expectation of the number of errors can be expressed as

$$
Q=\sum_{i=1}^{m_{c}} i \frac{\left(\begin{array}{c}
m_{c} \\
i
\end{array}\right)}{P-1}
$$

where $P-1$ incorrect position probability is $\frac{1}{P-1}$ and $\left(\begin{array}{c}n \\ m\end{array}\right)=$ $\frac{n !}{m !(n-m) !}$. Thus the BER of mapped bits is calculated as

$$
P_{\text {ecim }}=\frac{Q}{m_{c}} P_{e d},
$$

the BER for modulated bits can be given by

$$
P_{e m}=P_{e}\left(1-P_{e d}\right)+0.5 P_{e d},
$$

where the $P_{e}$ is bit error probability of the DCSK, and the probability of wrong PPM position detection is $50 \%$ when error probability of modulating bits is wrong, therefore the error probability of total system is expressed by

$$
P_{s y s}=\frac{m_{c}}{m_{c}+1} P_{e c i m}+\frac{1}{m_{c}+1} P_{e m}
$$

\section{B. Derivation of $P_{e d}$}

Assuming that both the modulating symbol $b_{l}=+1$ and $a_{l}=\hat{m}$ (it selects position index for PPM) are transmitted. Thus the mean and the variance of $I_{m}$ and $I_{m}^{\prime}$ are calculated by, respectively

$$
\begin{aligned}
\mu_{1} & =\mathrm{E}\left\{\mathrm{I}_{\mathrm{m}}\right\}=\frac{\sum_{l=1}^{L} \alpha_{l}^{2} E_{s}}{2}, \quad \mu_{2}=\mathrm{E}\left\{\mathrm{I}_{\mathrm{m}}^{\prime}\right\}=0, \\
\sigma_{1}^{2} & =\sum_{l=1}^{L} \operatorname{Var}\left\{\mathrm{I}_{\mathrm{m}}\right\}=\frac{\sum_{l=1}^{L} \alpha_{l}^{2} E_{s} N_{0}}{2}+\frac{N_{0}^{2} R}{4}, \\
\sigma_{2}^{2} & =\operatorname{Var}\left\{\mathrm{I}_{\mathrm{m}}^{\prime}\right\}=\frac{\sum_{l=1}^{L} \alpha_{l}^{2} E_{s} N_{0}}{4}+\frac{N_{0}^{2} R}{4} \\
& =E_{s} N_{0} \underbrace{\left.\sum_{l=1}^{\frac{\sum_{l=1}^{L} \alpha_{l}^{2}}{4}+\frac{R}{4 r_{s}}}\right)}_{\lambda}
\end{aligned}
$$

where $E_{s}=2 R \mathrm{E}\left\{\mathrm{x}^{2}\right\}$ is symbol energy of PPM-DCSK, $r_{s}=\sum_{l=1}^{L} \alpha_{l}^{2} E_{s} / N_{0}$ is signal-to-noise ratio, $\mathrm{E}\{\bullet\}$ is the expectation operator, and $\operatorname{Var}\{\bullet\}$ is the variance operator, then the random variables $\left|I_{m}\right|$ and $\left|I_{m}^{\prime}\right|$ follow identical folded normal distribution, thus the probability density function of 
$\left|I_{m}\right|$ and cumulative distribution function of $\left|I_{m}^{\prime}\right|$ are calculated respectively as

$$
\begin{gathered}
f_{\left|I_{m}\right|}(y)=\frac{1}{\sqrt{2 \pi \sigma_{\left|I_{m}\right|}^{2}}}\left\{e^{-\frac{\left(y-\mu_{\left|I_{m}\right|}\right)^{2}}{2 \sigma_{\left|I_{m}\right|}^{2}}}+e^{-\frac{\left(y+\mu_{\left|I_{m}\right|}\right)^{2}}{2 \sigma_{\left|I_{m}\right|}^{2}}}\right\}, \\
F_{\left|I_{m}^{\prime}\right|}(y)=\operatorname{erf}\left(\frac{y}{\sqrt{2 \sigma_{2}^{2}}}\right) .
\end{gathered}
$$

The $\mu_{\left|I_{m}\right|}$ and $\sigma_{\left|I_{m}\right|}^{2}$ are the mean and variance of the $\left|I_{m}\right|$ which can be expressed as

$$
\mu_{\left|I_{m}\right|}=\sqrt{\frac{2 \sigma_{1}^{2}}{\pi}} e^{-\frac{\mu_{1}^{2}}{2 \sigma_{1}^{2}}}-\mu_{1} \operatorname{erf}\left(\frac{-\mu_{1}}{\sqrt{2 \sigma_{1}^{2}}}\right)=\sqrt{E_{s} N_{0}} \gamma
$$

where

$$
\gamma=\sqrt{\frac{1}{2 \pi}+\frac{R}{4 \pi r_{s}}} e^{-\left(\frac{1}{r_{s}+\frac{2 R}{r_{s}^{2}}}\right)}-\frac{\sqrt{r_{s}}}{2} \operatorname{erf}\left(-\sqrt{\frac{1}{\frac{4}{r_{s}}+\frac{2 R}{r_{s}^{2}}}}\right),
$$

here and

$$
\begin{aligned}
& \sigma_{\left|I_{m}\right|}^{2}=\mu_{1}^{2}+\sigma_{1}^{2}-\mu_{\left|I_{m}\right|}^{2} \\
& =E_{s} N_{0} \underbrace{\left(\frac{r_{s}}{4}+\frac{1}{2}+\frac{R}{4 r_{s}}-\gamma^{2}\right)}_{\rho}
\end{aligned}
$$

Assume that $X_{1}=\max \left\{\left|I_{m}\right|\right\}, m=1,2, ., P-1$. And the position of PPM detection error probability is calculated by,

$$
\begin{aligned}
P_{e d} & =1-P_{r}\{Y \geq X\} \\
& =\int_{0}^{\infty}\left[1-P_{r}\{y \geq X\}\right] f_{\left|I_{m}\right|}(y) \mathrm{d} y \\
& =\frac{1}{\sqrt{2 \pi \sigma_{\left|I_{m}\right|}^{2}}} \int_{0}^{\infty}\left[1-\left[\operatorname{erf}\left(\frac{y}{\sqrt{2 \sigma_{2}^{2}}}\right)\right]^{P-1}\right] \\
& \times\left\{e^{-\frac{\left(y-\mu_{\left|I_{m}\right|}\right)^{2}}{2 \sigma_{\left|I_{m}\right|}^{2}}}+e^{-\frac{\left(y+\mu_{\mid I m}\right)^{2}}{2 \sigma_{|I m|}^{2}}}\right\} \mathrm{d} y .
\end{aligned}
$$

Let $\mu=\frac{y}{\sqrt{E_{s} N_{0}}}$, then the position detection error probability is derived as

$$
\begin{aligned}
& P_{e d}=\frac{1}{\sqrt{2 \pi \rho}} \int_{0}^{\infty}\left[1-\left[\operatorname{erf}\left(\frac{\mu}{\sqrt{2 \lambda}}\right)\right]^{P-1}\right] \\
& \times\left\{e^{-\frac{(y-\gamma)^{2}}{2 \rho}}+e^{-\frac{(y+\gamma)^{2}}{2 \rho}}\right\} \mathrm{d} \mu,
\end{aligned}
$$

$P_{e}$ is bit error probability of the DCSK which can be calculated as

$$
P_{e}=\frac{1}{2} \operatorname{erfc}\left(\left[\frac{4}{r_{s}}+\frac{2 R}{r_{s}^{2}}\right]^{-\frac{1}{2}}\right)
$$

total system Finally, substituting these above into (10), we obtain the instantaneous BER of PPM-DCSK over Rayleigh fading channel, and the multipath Rayleigh fading channel can be given as

$$
P_{\text {mul }}=\int_{0}^{\infty} P_{e} \cdot f\left(r_{s}\right) \mathrm{d} r_{s}
$$

where $f\left(r_{s}\right)$ is the PDF of $r_{s}$ which can be found in [5].

\section{SIMULATION RESULTS AND DISCUSSION}

In this section, simulations are carried out to evaluate the performance of the proposed system over AWGN and multipath Rayleigh fading channels. These results are then contrasted with the theoretical results derived in previous analysis on both AWGN and multipath Rayleigh fading channel. In all figures, $S F$ represents the spreading factor and $m_{c}$ is the number bits mapped into one position of the PPM modulation. In multipath Rayleigh fading channel, three paths $L=3$, are considered, having equal average power gain $\mathrm{E}\left[\alpha_{1}^{2}\right]=\mathrm{E}\left[\alpha_{2}^{2}\right]=\mathrm{E}\left[\alpha_{3}^{2}\right]=1 / 3$, with path delays $\tau_{1}=0, \tau_{2}=1, \tau_{3}=2$. To quickly illustrate, $m_{c}$ in PPMDCSK means modulated bits number of PPM parts and the whole number of modulated bits is $m_{c}+1$ while the whole number of M-DCSK is only $m_{c}$.

The analytical and simulated results for PPM-DCSK are shown in Fig. 3, where the analytical results match the simulations at high SNR. As can be also seen, BER always decreases as $m_{c}$ increases and as $S F$ decreases in AWGN while less influence in multipath fading. The reason for this behavior is that more bits in the same symbol energy are transmitted with increasing $m_{c}$, which means each transmitted bit needs less energy. Fig. 4 shows performance comparisons of PPM-DCSK, CIM-DCSK, CCI-DCSK and M-DCSK over AWGN channel. Note that the PPM-DCSK exhibits lower BER than the others, moreover the BER of M-DCSK increases as $m_{c}$ increases, while PPM-DCSK decreases as $m_{c}$ increases. The gain of PPM-DCSK over M-DCSK is at least about $4 \mathrm{~dB}$ as $m_{c}$ increase, and over CIM-DCSK and CCI-DCSK is about $0.3 \mathrm{~dB}, 2.5 \mathrm{~dB}$ at $10^{-4}$. The performance of PPMDCSK is significantly improved over M-DCSK. As shown in Fig. 5, BER performance of PPM-DCSK on multipath fading channel outperforms those of the other schemes when $S F=64$. All schemes have similar increasing BER behavior, PPM-DCSK has less increasing tendency then others when $m_{c}$ increasing. Note the good performance at high $m_{c}$ of the proposed PPM-DCSK. Fig. 6 shows BER performance with the modulated bits number of these schemes over AWGN and multipath Rayleigh fading channels. The performance of M-DCSK increases monotonously as $m_{c}$ increases. As $m_{c}$ increases, performance of PPM-DCSK, CIM-DCSK and CCIDCSK increases in fading but decreases in AWGN.

\section{Conclunsions}

In this letter, a novel hybrid modulation scheme combining PPM and DCSK is considered. The scheme avoids the problem of distinguishing inaccurately the constellation points in $M$ DCSK systems especially in large values of $M$. In the proposed system, information bearing signal is carried by PPM, which carries both DCSK modulated bit and extra red $m_{c}$ 


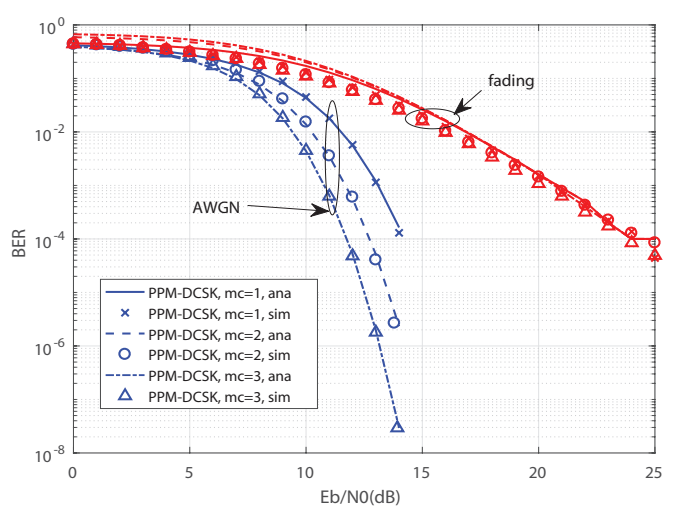

Fig. 3. Simulation and analytical BER of PPM-DCSK system over AWGN and Rayleigh multipath fading channel with spreading factor $S F=64$, the PPM modulated bits is $m_{c}$ and $P=2^{m_{c}}$.

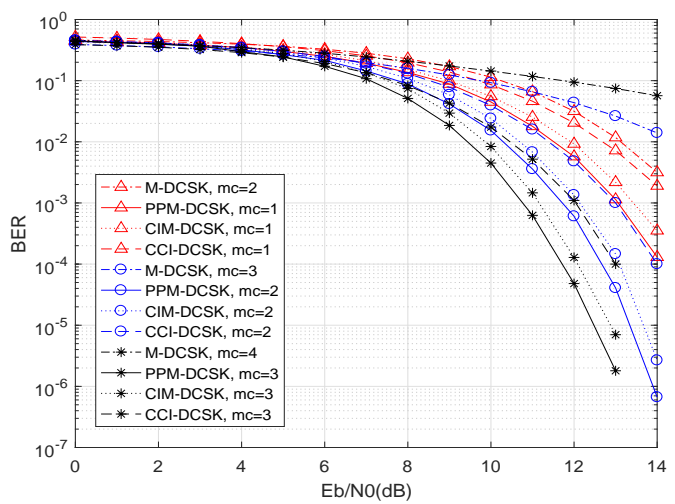

Fig. 4. Performance comparisons of PPM-DCSK, CIM-DCSK, CCI-DCSK, M-DCSK over AWGN channel $S F=64$.

bits by position modulation. The theoretical BER expression were derived and validated by simulations. By comparing the BER performance with conventional M-DCSK, CIM-DCSK and CCI-DCSK, the numerical results show that the proposed hybrid PPM-DCSK system has superior BER performance over AWGN and multipath Rayleigh fading channel.

\section{REFERENCES}

[1] Y. Tan, W. Xu, T. Huang and L. Wang, "A Multilevel Code Shifted Differential Chaos Shift Keying Scheme with Code Index Modulation," IEEE Trans. Circuits Syst. II, 2017, DOI: 10.1109/TCSII.2017.2764916.

[2] W. Xu, Y. Tan, F. C. M. Lau, and G. Kolumban, "Design and Optimization of Differential Chaos Shift Keying Scheme with Code Index Modulation," IEEE Trans. Commun., vol. 66, no. 5, pp. 19701980, Mar. 2018.

[3] G. Kolumban, B. Vizvari, W. Schwarz, and A. Abel, "Differential chaos shift keying: A robust coding for chaos communication," in Proc. NDES, Seville, Spain, 1996, pp. 87-92.

[4] M. Dawa, G. Kaddoum, and Z. Sattar, "A Generalized Lower Bound on the Bit Error Rate of DCSK Systems Over Multi-Path Rayleigh Fading Channels," IEEE Trans. Circuits Syst. II, vol. 65, no. 3, pp. 321-325, Mar. 2018.

[5] G. Cheng, L. Wang, W. Xu, and G. Chen, "Carrier Index Differential Chaos Shift Keying Modulation," IEEE Trans. Circuits Syst. II, vol. 64, no. 8, pp. 907-911, Aug. 2017.

[6] M. Herceg, G. Kaddoum, D. Vranjes, and E. Soujeri, "Permutation Index DCSK Modulation Technique for Secure Multi-User High-DataRate Communication Systems," IEEE. Trans. Veh. Technol., vol. 67, no. 4, pp. 2997-3011, Apr. 2018.

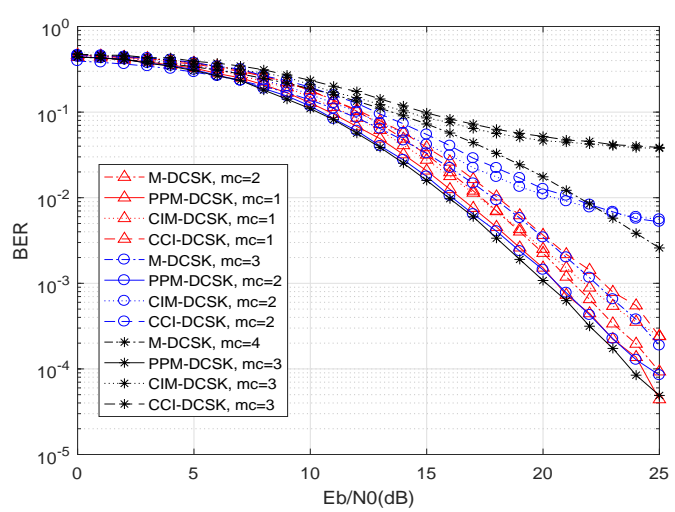

Fig. 5. BER performance comparisons of PPM-DCSK, CIM-DCSK, CCIDCSK, M-DCSK over Rayleigh multipath fading channel with $S F=64$, $L=3$.

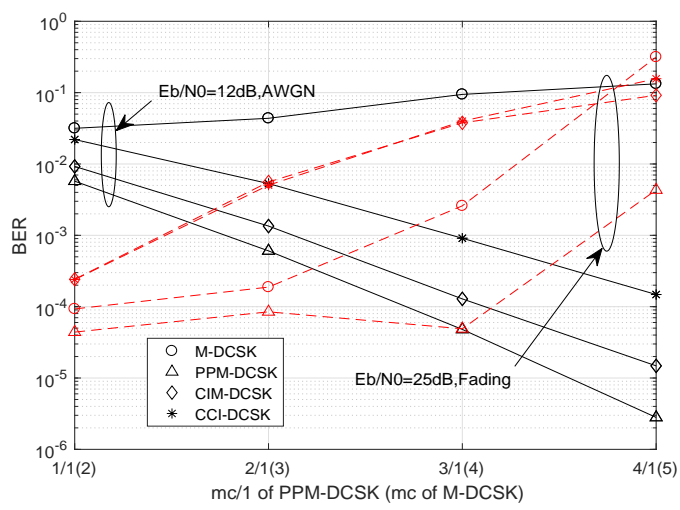

Fig. 6. Effect of the number of modulated bits on BER at PPM-DCSK, CIMDCSK, CCI-DCSK, M-DCSK over AWGN and Rayleigh multipath fading channel with $L=3, S F=64$.

[7] M. Herceg, D. Vranjes, G. Kaddoum, and E. Soujeri, "Commutation Code Index DCSK Modulation Technique for High-Data-Rate Communication Systems," IEEE Trans. Circuits Syst. II, 2018, DOI: 10.1109/TCSII.2018.2817930.

[8] G. Kaddoum, M. F. A. Ahmed, and Y. Nijsure, "Code Index Modulation: A High Data Rate and Energy Efficient Communication System," IEEE Commun. lett., vol. 19, no. 2, pp. 7000-7009, Feb. 2015.

[9] G. Kaddoum, Y. Nijsure, and H. Tran, "Generalized Code Index Modulation Technique for High-Data-Rate Communication Systems," IEEE. Trans. Veh. Technol., vol. 659, no. 9, pp. 175-178, Sept. 2016.

[10] L. Wang, G. Cai, and G. Chen, "Design and performance analysis of a new multiresolution M-ary differential chaos shift keying communication system," IEEE Trans. Wireless Commun.", vol. 14, no. 9, pp. 5197-5208, Sept. 2015.

[11] L. Bosotti, and G. Pirani, "A PAM-PPM signalling format in optical fiber digital communications," Opt. Quantum Electron., vol. 11, no. 1, pp. 71-86, Jan. 1979.

[12] Y. Dai, and X. Dong, "Hybrid PPM-BPSK for Transmitted Reference Pulse Cluster Systems in UWB and 60-GHz Channels," IEEE Trans. Wireless Commun., vol. 3, no. 6, pp. 657-660, Dec. 2014. 